arXiv:0804.3229

YITP-08-28

TU-816

KUNS-2136

IFT-UAM/CSIC-08-22

\title{
Non-perturbative moduli superpotential with positive exponents
}

\author{
Hiroyuki Abe ${ }^{1, *}$ Tetsutaro Higaki ${ }^{2, \dagger}$ Tatsuo Kobayashi ${ }^{3, \ddagger}$ and Osamu Seto ${ }^{4, \S}$ \\ ${ }^{1}$ Yukawa Institute for Theoretical Physics, Kyoto University, Kyoto 606-8502, Japan \\ ${ }^{2}$ Department of Physics, Tohoku University, Sendai 980-8578, Japan \\ ${ }^{3}$ Department of Physics, Kyoto University, Kyoto 606-8502, Japan \\ ${ }^{4}$ Instituto de Física Teórica UAM/CSIC, \\ Universidad Autónoma de Madrid, Cantoblanco, Madrid 28049, Spain
}

\begin{abstract}
We study non-perturbative moduli superpotentials with positive exponents, i.e. the form like $A e^{a T}$ with a positive constant $a$ and the modulus $T$. These effects can be generated, e.g., by D-branes which have negative RR charge of lower dimensional Dbrane. The scalar potentials including such terms have a quite rich structure. There are several local minima with different potential energies and a high barrier, whose height is of $\mathcal{O}\left(M_{p}^{4}\right)$. We discuss their implications from the viewpoints of cosmology and particle phenomenology, e.g. the realization of inflation models, avoiding the overshooting problem. This type of potentials would be useful to realize the inflation and low-energy supersymmetry breaking.
\end{abstract}

\footnotetext{
*E-mail address: abe@yukawa.kyoto-u.ac.jp

†E-mail address: tetsu@tuhep.phys.tohoku.ac.jp

${ }^{\ddagger}$ E-mail address: kobayash@gauge.scphys.kyoto-u.ac.jp

§E-mail address: osamu.seto@uam.es
} 


\section{Introduction}

Moduli fields play an important role in string phenomenology and cosmology. Several couplings in 4D low-energy effective field theory are given as functions of vacuum expectation values (VEVs) of moduli. Thus, we need a stabilization mechanism of moduli. However, some of moduli fields $T$ have a flat potential perturbatively, while others may be stabilized by non-trivial background such as flux compactification [1, 2]. Non-perturbative effects are assumed to stabilize such moduli. The form of non-perturbative terms in superpotential behaves like $e^{-a T}$, where $T$ is a modulus field and $a$ is a positive constant, and such terms would be induced by gaugino condensation and/or stringy non-perturbative effects. Such a potential may generate a hierarchically small energy scale compared with the Planck scale $M_{p}=2.4 \times 10^{18} \mathrm{GeV}$. That may be relevant to supersymmetry breaking and/or cosmological aspects, e.g. inflation models [3].

The bumps generated by the above terms $e^{-a T}$ are not high in several models of moduli stabilization, in particular, in models leading to low-energy supersymmetry breaking. That may lead to problems. For example, a simple model has a local minimum leading to a finite $\mathrm{VEV}$ of $T$ and the runaway vacuum, which is the minimum corresponding to $T \rightarrow \infty$, and there is a low bump between them. In such a model, we need fine-tuning of initial conditions in order not to overshoot the minimum with a finite VEV of modulus [4]. Also, such a low bump may have a problem from the viewpoint of inflation. Suppose that the inflaton $Z$ is different from $T$. We need the positive vacuum energy deriving inflation and it would be higher than the above bump. Then, the modulus would run away to infinity during the inflation. Similarly, finite temperature effects may also destabilize the modulus [5]. Furthermore, if the modulus $T$ is the inflaton, it seems difficult to realize the inflation and low-energy supersymmetry breaking in a simple model [6, 7].

In Ref. [8], it was pointed out that non-perturbative terms with positive exponents like $e^{a T}$, where $a>0$, can be induced in string-derived effective supergravity theory. Suppose that the gauge kinetic function $f$ is written as $f=m S-w T$ with $m, w>0$ and $S$ is stabilized with a heavy mass of $\mathcal{O}\left(M_{p}\right)$ by flux compactification. Then, the gaugino condensation of such a sector induces a non-perturbative term like $e^{a T}$ for the light modulus $T$. Some aspects of such a term, e.g. moduli stabilization and supersymmetry breaking, have been in Ref. [8].11 Here we study more about non-perturbative terms with positive exponents like $e^{a T}(a>0)$. They could generate high barriers in the scalar potential, whose heights would be of $\mathcal{O}\left(M_{p}^{4}\right)$. We study implications of such terms from the viewpoints of cosmology and particle phenomenology, in particular, the overshooting problem, realization of inflation models and low-energy supersymmetry breaking.

This paper is organized as follows. In section 2, we explain how non-perturbative terms with positive exponents like $e^{a T}$ can be generated in the superpotential, and study the form of scalar potential. In section 3, we study their implications on cosmology and particle phenomenology. In section 4, we apply to the racetrack inflation. Section 5 is devoted to conclusion and discussion.

\footnotetext{
${ }^{1}$ See also Refs. 9, 10. In particular, Ref. [10] has studied realization of the model solving the finetuning problem in the minimal supersymmetric standard model [11].
} 


\section{Non-perturbative moduli superpotential with pos- itive exponents}

For concreteness, we consider a supergravity model, which could be derived from type IIB superstring theory as its low-energy effective theory, although our supergravity model might be derived from other types of superstring theories such as type IIA superstring theory and heterotic string/M theory 2 In well-known Calabi-Yau models, there are three types of closed string moduli fields, the dilaton $S$, the Kähler (volume) moduli and complex structure (shape) moduli $U_{\alpha}$. For simplicity, we consider the model with a single Kähler modulus $T$, but extensions to models with several Kähler moduli are straightforward. Following Ref. [2], we assume that the dilaton $S$ and complex structure moduli $U_{\alpha}$ are stabilized by flux-induced superpotential $W_{\text {flux }}\left(S, U_{\alpha}\right)$ [12]. That implies that those moduli fields have heavy masses of $\mathcal{O}\left(M_{p}\right)$. Here and hereafter we use the unit that $M_{p}=1$.

In order to stabilize the remaining light modulus $T$, one often assumes the gaugino condensation in the hidden sector with the gauge kinetic function $f_{a}$, which induces the following term in the superpotential,

$$
W_{\mathrm{np}}=A e^{-\frac{2 \pi}{N_{a}} f_{a}},
$$

where $A=\mathcal{O}\left(M_{p}^{3}\right)$ and we have assumed that the hidden sector is described by a $\mathcal{N}=1$ pure super Yang-Mills (SYM) theory of $S U\left(N_{a}\right)$. Note that we use the normalization of the holomorphic gauge kinetic function $f_{a}$ such that the gauge coupling $g_{a}$ at cut off scale is obtained as

$$
\frac{4 \pi}{g_{a}^{2}}=R e\left(f_{a}\right)
$$

except non-holomorphic terms from $\sigma$-model anomalies. Thus, in the simple model with the holomorphic gauge kinetic function at tree level $f_{a}=T$ like the gauge sector on D7-brane 3 , the gaugino condensation induces 4

$$
W_{\mathrm{np}}=A e^{-\frac{2 \pi}{N_{a}} T} .
$$

However, the gauge kinetic function is written as a linear combination of two or more moduli in several string theories like heterotic string/M theory [14] and type II string theories with magnetized D-branes and/or intersecting D-branes [13, 15, 16, 17]. For example, a stack of magnetized D7-branes has the following gauge kinetic functions,

$$
f_{a}=m_{a} S+w_{a} T
$$

where $m_{a}$ and $w_{a}$ correspond to RR charges of D3-brane and one of D7-brane respectively, which are carried by the single magnetized D7-brane. On the magnetized D7-brane, they

\footnotetext{
${ }^{2}$ Definitions of modulus in type IIA and IIB superstring theories and heterotic string/M theory are different from each other.

${ }^{3}$ Hereafter we use a symbol of $f_{a}$ as a holomorphic gauge coupling at string tree level.

${ }^{4}$ From this equation, a factor of $A$ can include VEVs of complex structure moduli $U_{\alpha}$, whose effects come from threshold corrections to gauge coupling by heavy mode in $\mathcal{N}=2$ SUSY sector of open string. For the (non-)perturbative corrections to gauge coupling, see Ref. [13.
} 
are positive rational numbers determined by magnetic fluxes and winding numbers 5 . Similar gauge kinetic functions can be derived from other string theories including heterotic string/M theory and type IIA superstring theory. When the gaugino condensation happens in the $\mathcal{N}=1$ pure SYM of $S U\left(N_{a}\right)$ with this gauge kinetic function (4), the following term in the superpotential is induced,

$$
W_{\mathrm{np}}=A e^{-\frac{2 \pi}{N_{a}}\left(m_{a} S+w_{a} T\right)} .
$$

We assume that the dilaton $S$ is already stabilized with the mass of $\mathcal{O}\left(M_{p}\right)$ by the flux compactification. Thus, here we replace $S$ by its VEV $S_{0} 6$ Then, the superpotential reduces to the form $A^{\prime} e^{-a T}$ with $A^{\prime}=A e^{-\frac{2 \pi}{N_{a}} m_{a} S_{0}}$ and $a=\frac{2 \pi}{N_{a}} w_{a}$, and its form is almost the same as the superpotential (3). However, the coefficient $A^{\prime}$ can be hierarchically suppressed compared with $M_{p}^{3}$, because of the factor $e^{-\frac{2 \pi}{N_{a}} m_{a} S_{0}}$.

On the other hand, the following form of gauge kinetic function

$$
f_{a}=m_{a} S-w_{a} T
$$

with positive rational numbers $m_{a}$ and $w_{a}$ can also be derived from supersymmetric magnetized D9-brane which carries negative RR charge of D7-brane [19] 7, as well as heterotic string/M theory and type IIA superstring theory, when $m_{a} R e(S)-w_{a} \operatorname{Re}(T)>$ 08 As the magnetized D7-brane, $m_{a}$ and $-w_{a}$ correspond to RR charge of D3-brane and one of D7-brane respectively, which are carried by the single magnetized D9-brane. The gaugino condensation in the hidden sector of $\mathcal{N}=1$ pure SYM of $S U\left(N_{a}\right)$ with this gauge coupling induces the following non-perturbative term in the superpotential 9 ,

$$
W_{\mathrm{np}}=A e^{-\frac{2 \pi}{N_{a}}\left(m_{a} S_{0}-w_{a} T\right)} .
$$

Here, we have assumed that the dilaton is stabilized with a heavy mass of $\mathcal{O}\left(M_{p}\right)$ and replaced $S$ by its VEV $S_{0}$. The superpotential (7) corresponds to the form $A^{\prime} e^{a T}$ with the

\footnotetext{
${ }^{5}$ The form of gauge couplings in this paper can be found in compactifications of toroidal orbifold at least. However, in generic Calabi-Yau compactifications this changes due to the geometric curvature terms. For example, $\mathcal{O}\left(\mathcal{R}^{2}\right)$ terms exist in $m_{a}$ of a gauge coupling on the (magnetized) D7-brane [18]. Then we may have negative $m_{a}$ of it as magnetized D9-branes which have negative D3-brane charges 19. At any rate, we will mention the case which is independent of geometric curvature terms.

6 Such replacement by $S_{0}$ is valid in the case that the dilaton mass is much larger than the mass of Kähler modulus $T$ [20, 21, 22]. (See e.g. Ref. 23] for the model that both $S$ and $T$ are light moduli.) This condition is satisfied with the cases that we study.

${ }^{7}$ This means that we have (almost) vanishing Fayet-Iliopoulos D-term on the magnetized D9-brane. For example, in $T^{6} /\left(Z_{2} \times Z_{2}\right)$ orientifold $\left(h_{1,1}^{(+)}\right.$untwist $\left.=3\right)$, the condition is proportial to the equation, e.g., $D \propto \frac{1}{m \operatorname{Re}(S)}+\frac{1}{w_{i} \operatorname{Re}\left(T_{i}\right)}+\frac{1}{w_{j} \operatorname{Re}\left(T_{j}\right)}-\frac{1}{w_{k} \operatorname{Re}\left(T_{k}\right)} \simeq 0$ with $\forall w_{i}, m>0$, while a holomorphic gauge coupling on the magnetized D-brane is given by $f=m S+w^{i} T_{i}+w^{j} T_{j}-w^{k} T_{k}$. Here $i, j, k=1,2,3$, $i \neq j \neq k \neq i$ and we omitted the contributions of matter fields.

${ }^{8}$ In D-brane systems a gauge coupling at tree level on a D-brane is given by a VEV of linear combination of moduli, which means effective volume wrapped by the D-brane. Especially, in type IIB O3/O7 system tree level gauge coupling would be reliable as long as $\operatorname{Re}(T) / \operatorname{Re}(S)=R^{4}>1$, where $R$ is a radius of compactification normalized by string length $\alpha^{1 / 2}$ in string frame. In heterotic case, a (linear) combination of moduli appears at 1-loop (or next $\kappa_{11}^{2 / 3}$ ) order.

${ }^{9}$ For gauge couplings $f_{a}^{k}=m S+w_{a}^{i} T_{i}+w_{a}^{j} T_{j}-w_{a}^{k} T_{k}$, where $a$ represents a label for stacks of the magnetized D9-branes, $i, j, k=1,2,3$ and $i \neq j \neq k \neq i$, it is sufficient for us to have a superpotential, e.g., $W=A_{1} e^{-a_{1} f_{1}^{1}}+A_{2} e^{-a_{2} f_{2}^{2}}+A_{3} e^{-a_{3} f_{3}^{3}}$, which prevents each modulus from running away to infinity, though in $T^{6} /\left(Z_{2} \times Z_{2}\right)$ orientifold, studies for the stabilization of open string moduli are important [24].
} 
positive exponent $a=\frac{2 \pi w_{a}}{N_{a}}$, and that leads to the moduli potential quite different from one only with negative exponents, i.e. $A^{\prime} e^{-a T}$. The region around $w_{a} T=m_{a} S_{0}$ corresponds to the strong gauge coupling region, where it would not be reliable to consider only the term $A e^{-\frac{2 \pi}{N_{a}} f_{a}}$ in the superpotential. The strong gauge coupling region would correspond to $4 \pi / g^{2}=\operatorname{Re}\left(f_{a}\right) \lesssim 1$, i.e.,

$$
m_{a} \operatorname{Re}\left(S_{0}\right)-w_{a} \operatorname{Re}(T) \lesssim 1
$$

Outside of this region (8) the above superpotential (17) is reliable.

The F-term scalar potential $V_{F}$ is written as

$$
V_{F}=e^{K}\left[\left|D_{T} W\right|^{2} K^{T \bar{T}}-3|W|^{2}\right]
$$

with $D_{T} W \equiv K_{T} W+W_{T}$ and

$$
K=-3 \ln (T+\bar{T})
$$

where $K_{T}$ and $W_{T}$ denote first derivatives of $K$ and $W$ by $T$, respectively. The gravitino mass $m_{3 / 2}$ is obtained as $m_{3 / 2}^{2}=e^{K}|W|^{2}$. The scalar potential $V_{F}$ including the above superpotential (77) has a quite high barrier around $w_{a} \operatorname{Re}(T) \approx m_{a} \operatorname{Re}\left(S_{0}\right) 10$ and its reliable height is at least $V_{F} \sim|A|^{2} e^{-4 \pi / N_{a}}$, where we have estimated at $\operatorname{Re}\left(f_{a}\right) \sim 1$. Thus, this barrier height is almost of $\mathcal{O}\left(M_{p}^{4}\right)$ when $4 \pi / N_{a} \sim 1$. That has significant implications in cosmology and particle phenomenology. We shall study them in the next sections.

\section{Potential forms and their Implications}

Here, let us study implications of the following total superpotential,

$$
W_{\text {tot }}=W_{0}+\sum_{a} A_{a} e^{-\frac{2 \pi}{N_{a}}\left(m_{a} S_{0}+w_{a} T\right)}+\sum_{b} A_{b} e^{-\frac{2 \pi}{N_{b}}\left(m_{b} S_{0}-w_{b} T\right)},
$$

where ${ }^{\forall} A_{a}, A_{b}=\mathcal{O}\left(M_{p}^{3}\right)$. In particular, the third term is important. The first term corresponds to the VEV of the flux-induced superpotential $W_{\text {flux }}\left(S, U_{\alpha}\right)$ and/or nonperturbative terms including only the dilaton $S$, i.e., $A_{a} e^{-\frac{2 \pi}{N_{a}} m_{a} S_{0}}$.

We consider the corresponding F-term scalar potential $V_{F}$, which would have several local minima. Also we add the uplifting potential, $E /(T+\bar{T})^{n}$ following [2] and the total potential is obtained as

$$
V=V_{F}+\frac{E}{(T+\bar{T})^{n}} .
$$

Such uplifting potential can be generated by anti D3-brane [2] 11 We tune the constant $E$ such that one of local minima has a small positive vacuum energy, $V \simeq 10^{-120}$.

This potential $V$ has a quite rich structure. First of all, the potential $V$ as well as $V_{F}$ has a barrier with height of $\mathcal{O}\left(M_{p}^{4}\right)$ around $T \approx m_{b} S_{0} / w_{b}$. Such a high barrier would be useful to avoid the overshooting problem and destabilization due to inflation driving energy and finite temperature effects. Furthermore, this potential may have several local minima with hierarchically different potential energies. That would be useful to realize both inflation and low-energy supersymmetry breaking.

\footnotetext{
${ }^{10}$ Then we will need a condition that $m_{a}>w_{a}$ from the condition that $\operatorname{Re}(T) / \operatorname{Re}\left(S_{0}\right)>1$. For a detail, see Ref. 8 .

11 Similar uplifting is possible by spontaneous SUSY breaking sectors [25, 26].
} 


\subsection{Superpotential with a single term}

One of the simplest models is the model with the following total superpotential,

$$
W_{\text {tot }}=A e^{-\frac{2 \pi}{N}(m S-w T)} .
$$

This superpotential is R-symmetric. As shown in Ref.[27], the SUSY point $D_{T} W=0$ corresponds to a local maximum of the F-term scalar potential $V_{F}$ and it has a SUSY breaking local minimum at $\operatorname{Re}(T)=2 / a$, where $a=2 \pi w / N 12$ This local minimum always has a negative vacuum energy $V_{F}<0$. We need the uplifting term $E /(T+\bar{T})^{n}$ to realize a de Sitter vacuum. Then, we require $V=V_{F}+E /(T+\bar{T})^{n} \simeq 0$ and $\partial_{T} V=0.13$ These provide with the following condition,

$$
\frac{1}{3} a^{2} t^{2}+\frac{1}{3}(n-7) a t+(4-2 n)=0,
$$

where $t=2 R e(T)$. For example, for $n=2$, this condition is satisfied when

$$
2 R e(T)=\frac{5}{a}=\frac{5 N}{2 \pi w}
$$

and in this case the total scalar potential is given as

$$
V(t)=\frac{a\left|A^{\prime}\right|^{2}}{3 t^{2}}\left[e^{5}+e^{a t}(a t-6)\right],
$$

where $A^{\prime}=A e^{-2 \pi m S_{0} / N}$. For a small value of $\operatorname{Re}(T)$, corrections to the Kähler potential would be important. In order to realize the minimum with $\operatorname{Re}(T)=\mathcal{O}(4 \pi)$ from Eq. (15), we need $N / w=\mathcal{O}(10)-\mathcal{O}(100)$. At the minimum, SUSY is broken and the F-term of the modulus $T$,

$$
F^{T}=-e^{K / 2} K^{T \bar{T}} D_{T} W
$$

is evaluated as

$$
\frac{F^{T}}{T+\bar{T}}=-\frac{2}{3} m_{3 / 2}
$$

where the gravitino mass $m_{3 / 2}$ is given as $m_{3 / 2}=A^{\prime} e^{5 / 2} /(2 \operatorname{Re}(T))^{3 / 2}$. For $\operatorname{Re}(T)=\mathcal{O}(4 \pi)$, the F-term $F^{T}$ is sizable compared with the anomaly mediation. Similarly, for $n=3$, the above condition (14) is satisfied when

$$
2 R e(T)=\frac{2+\sqrt{10}}{a}=\frac{(2+\sqrt{10}) N}{2 \pi w},
$$

and the total scalar potential is given as

$$
V(t)=\frac{a\left|A^{\prime}\right|^{2}}{3 t^{3}}\left[2(\sqrt{10}-1) e^{2+\sqrt{10}}+e^{a t} a t(a t-6)\right] .
$$

The size of $F^{T} /(T+\bar{T})$ is of $\mathcal{O}\left(m_{3 / 2}\right)$, as the case with $n=2$.

\footnotetext{
12 A similar potential was discussed for twisted moduli [28].

${ }^{13}$ In order to obtain the minimum, the condition that $\frac{1}{2} a^{3} t^{3}-4 a^{2} t^{2}+\left(13-\frac{n(n+1)}{2}\right) a t+3(n(n+1)-6)>$ 0 must be satisfied, too.
} 
All of the above aspects are different form the superpotential $W_{\text {tot }}=A^{\prime} e^{-a T}$ with $a>0$, whose scalar potential has no local minimum. Thus, the total superpontential (13) gives the simplest model for modulus stabilization, that is, the superpotential with a single term. Also, this is the simplest model from the viewpoint to realize the modulus mediation. (See also Ref. [23].)

The real part of $T$ can be stabilized by this simple R-symmetric superpotential, but the potentials with and without the uplifting term do not include $\operatorname{Im}(T)$. This aspect may be important from the viewpoint of the QCD axion 14 (See e.g. Ref [29] and references therein.)

\subsection{Superpotential with two terms: the KKLT type and racetrack type}

Next, we consider models of the total superpotentials with only two terms. Among them, the KKLT type of the total superpotential,

$$
W_{\mathrm{tot}}=W_{0}+A e^{-\frac{2 \pi}{N}\left(m S_{0}+w T\right)}
$$

with $m \geq 0, w>0$ and the racetrack type,

$$
W_{\text {tot }}=A_{1} e^{-\frac{2 \pi}{N_{1}}\left(m_{1} S_{0}+w_{1} T\right)}+A_{2} e^{-\frac{2 \pi}{N_{2}}\left(m_{2} S_{0}+w_{2} T\right)},
$$

with $m_{a} \geq 0, w_{a}>0(a=1,2)$ are well-known. The corresponding F-term scalar potentials $V_{F}$ have local minima determined by $D_{T} W=0$, i.e.,

$$
\operatorname{Re}(T) \approx-\frac{m}{w} \operatorname{Re}\left(S_{0}\right)+\frac{N}{2 \pi w} \ln \left(A / W_{0}\right)
$$

for $\frac{2 \pi w}{N} \operatorname{Re}(T)=\mathcal{O}(10)$ in the KKLT type and

$$
\operatorname{Re}(T) \approx \frac{-m_{1} N_{2}+m_{2} N_{1}}{w_{1} N_{2}-w_{2} N_{1}} \operatorname{Re}\left(S_{0}\right)+\frac{1}{2 \pi\left(w_{1} / N_{1}-w_{2} / N_{2}\right)} \ln \left(\frac{A_{1} w_{1} N_{2}}{A_{2} w_{2} N_{1}}\right)
$$

for $\frac{2 \pi w_{a}}{N_{a}} \operatorname{Re}(T)=\mathcal{O}(10)(a=1,2)$ in the racetrack type, respectively. In both models, $\operatorname{Im}(T)$ is determined at local minima. These vacua remain even after we add the uplifting potential $E /(T+\bar{T})^{n}$ tuning $E$ such that these vacua have almost vanishing energies. Both models have the runaway behavior at $\operatorname{Re}(T) \rightarrow \infty$, and such runaway vacuum and local minima are separated by not high bumps. For example, the KKLT model has a low bump, whose height is of $\mathcal{O}\left(\left|W_{0}\right|^{2}\right)=\left(m_{3 / 2}^{2} M_{p}^{2}\right)$, when one tunes the constant $E$ in the uplifting potential such that the above local minimum has almost vanishing vacuum energy. Thus, the inflaton potential energy must be lower than the height of $\left(m_{3 / 2}^{2} M_{p}^{2}\right)$ to aviod the runaway behavior. Since it means $H_{\text {inf }}<m_{3 / 2}$ with $H_{\text {inf }}$ being the Hubble parameter during inflation [6], inflation of a very low energy scale or a very heavy gravitino mass is required. For such a low energy inflation, conceptually new flatness problem is reintroduced and phenomenologically the detection of tensor type perturbation is hopeless. On the other hand, very heavy gravitino is not desired from the viewpoint of weak scale

\footnotetext{
${ }^{14}$ The decay constant in this model would be of $\mathcal{O}\left(M_{p}\right)$ or order of the GUT scale. We would need some mechanism to lead to the cosmologically allowed window of the decay constant.
} 
supersymmetry as a solution to the hierarchy problem. Similarly, the racetrack model has a low bump between the local minimum and the runaway vacuum. Such forms of potentials would have problems, and one of them is the overshooting problem, that is, we need the fine-tuning of initial conditions for realizing the local minimum with a finite value of $T$. Otherwise, the value of $\operatorname{Re}(T)$ runs away to infinity, $\operatorname{Re}(T) \rightarrow \infty$. Furthermore, it would be difficult to realize inflation models in the above types of potentials. We will discuss this point in section 3.3.

Now, let us study other types of the total superpotentials with two terms,

$$
W_{\text {tot }}=W_{0}+A e^{-\frac{2 \pi}{N}\left(m S_{0}-w T\right)},
$$

with $m, w>0$ and

$$
W_{\text {tot }}=A_{1} e^{-\frac{2 \pi}{N_{1}}\left(m_{1} S_{0}+w_{1} T\right)}+A_{2} e^{-\frac{2 \pi}{N_{2}}\left(m_{2} S_{0}-w_{2} T\right)},
$$

with $m_{1} \geq 0, m_{2}, w_{a}>0(a=1,2)$. These may look similar to the KKLT type and the racetrack type of superpotentials. However, the second terms in both superpotentials have positive exponents for $T$ for certain regions of $\operatorname{Re}(T)$, and that leads to quite different features. The corresponding scalar potentials $V_{F}$ as well as $V$ have barriers around $w \operatorname{Re}(T) \approx m \operatorname{Re}\left(S_{0}\right)$ and $w_{2} \operatorname{Re}(T) \approx m_{2} \operatorname{Re}\left(S_{0}\right)$, respectively. They have local minima corresponding to $D_{T} W=0$, i.e.,

$$
\operatorname{Re}(T) \approx \frac{N}{2 \pi w} \ln \left(W_{0} / A\right)+\frac{m}{w} \operatorname{Re}\left(S_{0}\right),
$$

for $W_{\text {tot }}(\underline{25})$, and

$$
R e(T) \approx \frac{m_{2} N_{1}-m_{1} N_{2}}{w_{1} N_{2}+w_{2} N_{1}} \operatorname{Re}\left(S_{0}\right)+\frac{1}{2 \pi\left(w_{1} / N_{1}+w_{2} / N_{2}\right)} \ln \left(\frac{A_{1} w_{1} N_{2}}{A_{2} w_{2} N_{1}}\right),
$$

for $W_{\text {tot }}(26)$. Since obviously there is a barrier of $\mathcal{O}\left(M_{p}^{4}\right)$ for small $\operatorname{Re}(T)$ because of the Kähler potential $K=-3 \ln (T+\bar{T})$, the above local minima are surrounded by high barriers of $\mathcal{O}\left(M_{p}^{4}\right)$. Thus, the overshooting problem would be avoided, that is, we do not need the fine-tuning to realize the above local minima. This aspect would also be useful for realization of the inflation as we will discuss in section 3.3.

Finally, among the class of superpotentials with only two terms, let us consider the following,

$$
W_{\text {tot }}=A_{1} e^{-\frac{2 \pi}{N_{1}}\left(m_{1} S_{0}-w_{1} T\right)}+A_{2} e^{-\frac{2 \pi}{N_{2}}\left(m_{2} S_{0}-w_{2} T\right)},
$$

with $m_{a}, w_{a}>0(a=1,2)$, which satisfies $m_{1} / w_{1}<m_{2} / w_{2}, w_{1} / N_{1}>w_{2} / N_{2}$ and $m_{1} / N_{1}>$ $m_{2} / N_{2}$. We restrict to the region $0 \lesssim R e(T) \lesssim m_{1} R e\left(S_{0}\right) / w_{1}$. That looks similar to the racetrack superpotential, but the sign of exponents for $T$ is opposite. In this case, there is the local minimum,

$$
\operatorname{Re}(T) \approx \frac{m_{1} N_{2}-m_{2} N_{1}}{w_{1} N_{2}-w_{2} N_{1}} \operatorname{Re}\left(S_{0}\right)+\frac{-1}{2 \pi\left(w_{1} / N_{1}-w_{2} / N_{2}\right)} \ln \left(\frac{A_{1} w_{1} N_{2}}{A_{2} w_{2} N_{1}}\right) .
$$

Note that in this region of parameters the first term is positive.

In the superpotential with three or more terms, the corresponding scalar potential has a richer structure. There are several local minima with different potential energies in addition to the runaway vacuum and/or there is a high barrier, whose height would be of $\mathcal{O}\left(M_{p}^{4}\right)$. That would be useful, e.g. to realize the inflation and low-energy supersymmetry breaking. 


\subsection{Stability during inflation}

As discussed in the previous sections, section 3.1 and 3.2, the behavior of the total superpotential with the positive exponent term $A e^{a T}(a>0)$ is quite different from the total superpotential without such a term. The bump of the scalar potential without the positive exponent term would be much lower than the Planck scale. That would have several problems such as the overshooting problem and instability due to finite temperature effects and/or vacuum energy deriving inflation. On the other hand, the scalar potential with the positive exponent term would have a high barrier, whose reliable height is almost of $\mathcal{O}\left(M_{p}^{4}\right)$. That would be useful to avoid the above problems. Here, we consider the inflation model, where some field $Z$ other than the modulus $T$ plays a role as the inflaton.

Suppose that we have a supergravity inflation model with the inflaton $Z$, when the degree of freedom of the modulus $T$ is frozen (by hand). We write its potential as $V_{\text {inf }}(Z, \bar{Z})$, and its value during the inflation would be much higher than heights of the above bumps of the superpotential without the positive exponent term, e.g. $V_{\text {inf }}(Z, \bar{Z}) \gg\left|W_{0}\right|^{2}$ in (21). However, the inflation is difficult to be realized when we consider the modulus $T$ as the dynamical field, which will spoil original inflationary dynamics, e.g. the slow-roll condition. If the F-term is dominant in this inflation model, the total potential would behave like

$$
V(T, \bar{T}, Z, \bar{Z}) \sim V_{\text {modulus }}+\frac{V_{\text {inf }}(Z, \bar{Z})}{(T+\bar{T})^{3}}+\cdots,
$$

during the inflation, where $V_{\text {modulus }}$ denotes the scalar potential stabilizing $T$ when $V_{\text {inf }}(Z, \bar{Z})$ is absent. Note that $V_{\text {inf }}(Z, \bar{Z}) \gg\left|W_{0}\right|^{2}$. Then, $T$ runs away to infinity. If the D-term potential, $V_{D}=\frac{g^{2}}{2} D^{2}$ is dominant during the inflation and the $T$-dependence appears only through the gauge coupling $g^{2} \sim 1 /(T+\bar{T})$, the total potential would behave like

$$
V(T, \bar{T}, Z, \bar{Z}) \sim V_{\text {modulus }}+\frac{V_{\text {inf }}(Z, \bar{Z})}{(T+\bar{T})}+\cdots,
$$

during the inflation, and in this model $T$ runs away to infinity. Also, in other cases, it would be difficult to realize the inflation model of $Z$ for the dynamical $T$ with a low bump potential.

However, the situation is different when the total superpotential includes a positive exponent term, $A e^{-\frac{2 \pi}{N}\left(m S_{0}-w T\right)}$. The scalar potential $V_{\text {modulus }}$ has a high barrier around $\operatorname{Re}(T)=m \operatorname{Re}\left(S_{0}\right) / w$, and its height is of $\mathcal{O}\left(M_{p}^{4}\right)$. Thus, $\operatorname{Re}(T)$ does not run away to infinity during the inflation, when its initial value is smaller than the location of high barrier, that is, $\operatorname{Re}(T)$ would be stabilized at $e^{-\frac{2 \pi}{N}\left[m\left(S_{0}+\bar{S}_{0}\right)-w(T+\bar{T})\right]} \sim V_{\inf }(Z, \bar{Z})$ during the inflation and its mass would be larger than the inflation Hubble value by a factor $(2 \pi w / N) \operatorname{Re}\left(T_{\mathrm{inf}}\right)=\mathcal{O}(10) 15$ Hence, the positive exponent term would be useful to stabilize the modulus during the inflation if we have a supergravity inflation model with the inflation $Z$. In the next section, we consider the inflation model, where the modulus is the inflaton.

\footnotetext{
${ }^{15}$ The $\operatorname{Re}\left(T_{\mathrm{inf}}\right)$ means fixed value during inflation by $Z$ field and will satisfy an inequality that $\langle\operatorname{Re}(T)\rangle<\operatorname{Re}\left(T_{\mathrm{inf}}\right) \lesssim m \operatorname{Re}\left(S_{0}\right) / w$.
} 


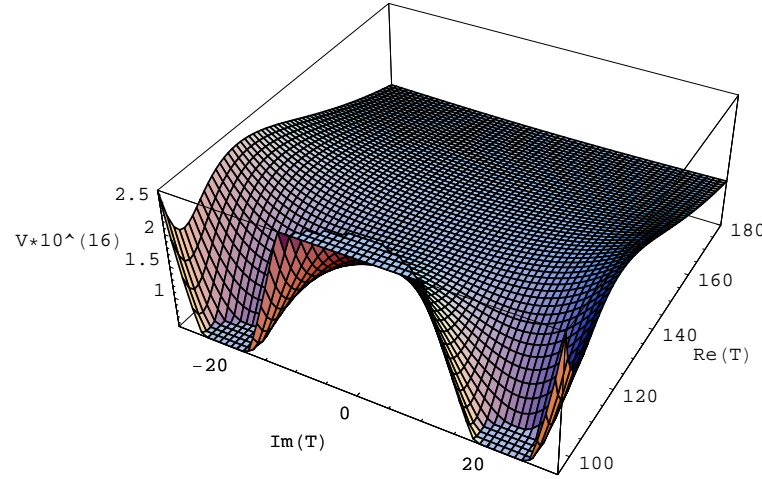

(a)

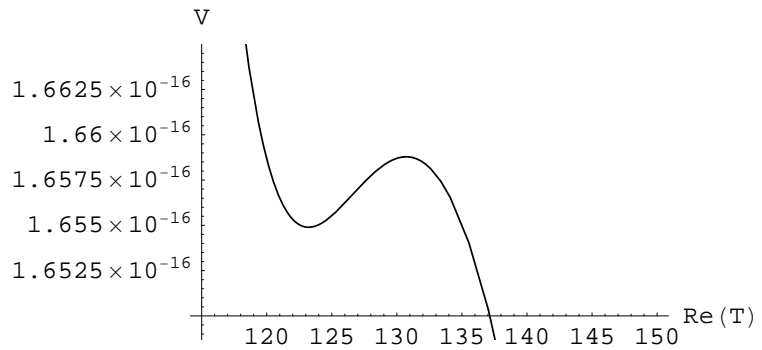

(b)

Figure 1: The graph (a) shows a racetrack type scalar potential derived from Eq. (33) with $A_{3}=0$ (rescaled by $10^{16}$ ). Inflation begins in a vicinity of the saddle point at $\operatorname{Re}(T)=$ 123.22, $\operatorname{Im}(T)=0$. The graph (b) shows a slice of the potential along $\operatorname{Im}(T)=0$.

\section{Application example: racetrack inflation}

Here, we show one of application examples of the superpotential (11), discussing the inflation model, where the modulus is the inflaton. We consider the following total superpotential,

$$
W_{\mathrm{tot}}=W_{0}+\sum_{a=1,2} A_{a} e^{-\frac{2 \pi}{N_{a}}\left(m_{a} S_{0}+w_{a} T\right)}+A_{3} e^{-\frac{2 \pi}{N_{3}}\left(m_{3} S_{0}-w_{3} T\right)},
$$

with $m_{1,2} \geq 0, m_{3}, w_{a}>0$ for $a=1,2,3$. When $A_{3}=0$, the above superpotential (33) corresponds to one in the racetrack inflation model [30]. (See also Ref. [31].) Actually, when we choose parameters appropriately, e.g. 30]

$$
\begin{aligned}
& \frac{N_{1}}{w_{1}}=100, \quad \frac{N_{2}}{w_{2}}=90, \quad W_{0}=-\frac{1}{25000}, \\
& A_{1} e^{-\frac{2 \pi}{N_{1}} m_{1} S_{0}}=\frac{1}{50}, \quad A_{2} e^{-\frac{2 \pi}{N_{2}} m_{2} S_{0}}=-\frac{35}{1000},
\end{aligned}
$$

there is a saddle point at $(\operatorname{Re}(T), \operatorname{Im}(T))=(123.22,0)$ and the two minima $(\operatorname{Re}(T), \operatorname{Im}(T))=$ $(96.130, \pm 22.146)$ for $A_{3}=0$. Around the saddle point, the slow-roll inflation can be realized. That is, at the saddle point, we obtain $\varepsilon=0$ and $\eta=-0.006097$. The slow-roll parameters $\varepsilon$ and $\eta$ are defined as

$$
\varepsilon \equiv \frac{M_{p}^{2}}{2} \frac{1}{V^{2}}\left(\frac{d V}{d \phi}\right)^{2}, \quad \eta \equiv M_{p}^{2} \frac{1}{V} \frac{d^{2} V}{d^{2} \phi},
$$

for the canonically normalized inflaton $\phi$.

Fig 1(a) shows the scalar potential around the saddle point for $A_{3}=0$, and the section along the direction $\operatorname{Im}(T)=0$ is shown in Fig.1 (b). The bump around $\operatorname{Re}(T) \approx 130$ is low and the modulus $\operatorname{Re}(T)$ may overshoot the saddle point and run away to infinity. 


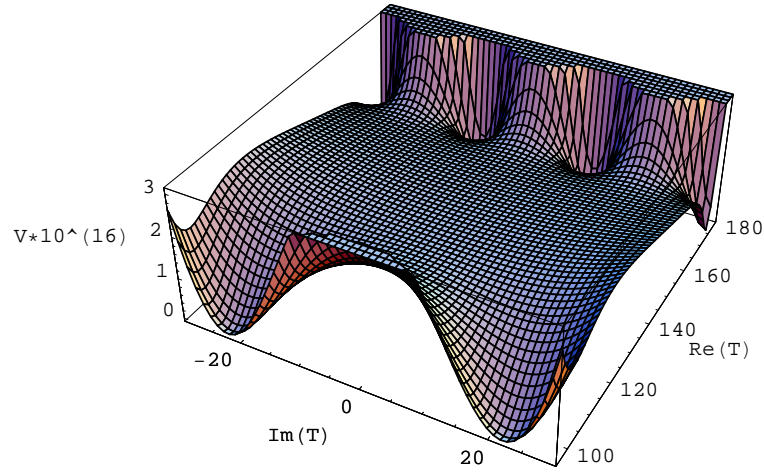

(a)

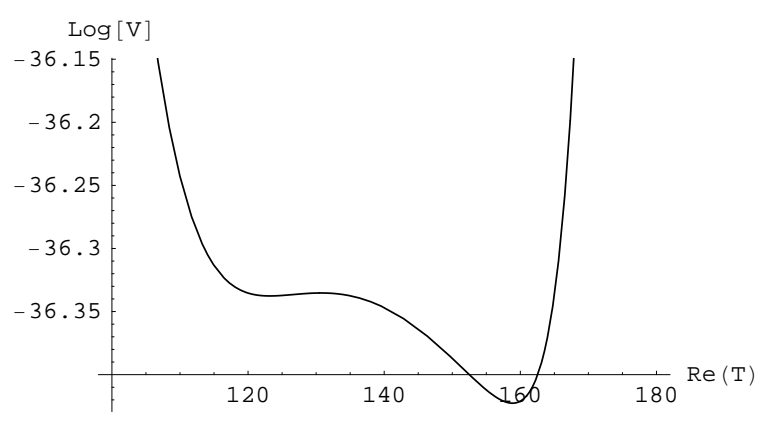

(b)

Figure 2: The graph (a) shows a racetrack type scalar potential derived from Eq. (33) with $A_{3}=1$ (rescaled by $10^{16}$ ). Inflation begins in a vicinity of the saddle point at $\operatorname{Re}(T)=123.22, \operatorname{Im}(T)=0$ and we can find new minima which are shallower than old ones around $\operatorname{Re}(T) \approx 173, \operatorname{Im}(T) \approx 10$ and high barrier around $\operatorname{Re}(T) \approx 210$. The graph (b) shows a slice of the natural logarithm of the potential along $\operatorname{Im}(T)=0$.

Now, we add the third term of the superpotential (33) such that it lifts up the runaway direction without violating the potential behavior around the saddle point. For example, when we tune the parameters as 16

$$
A_{3}=1, \quad m_{3} S_{0}=68.8 \pi, \quad w_{3}=1, \quad N_{3}=20,
$$

the scalar potential becomes as shown in Fig. 2. The height of the barrier around $\operatorname{Re}(T) \approx$ 210 is of $\mathcal{O}\left(M_{p}^{4}\right)$ and that would be helpful to avoid the overshooting problem. In addition, at the saddle point, we obtain $\varepsilon=0$ and $\eta=-0.006850$, that is, the potential behavior around the saddle point, which is important to realize the inflation, does not change. Indeed, before the inflaton rolls down to the minimum $(\operatorname{Re}(T), \operatorname{Im}(T))=(96.130,22.146)$, we can realize $N=130$ e-foldings when we use the initial conditions $(\operatorname{Re}(T), \operatorname{Im}(T))=$ $(123.22,0.1)$, which is a vicinity of the saddle point. This number of e-folds is almost the same as one obtained in Ref. [30]. Thus, adding the superpotential term with positive exponent is useful to lift up the runaway direction.

Furthermore, we could construct other racetrack inflation models with the following superpotential,

$$
W=W_{0}+A e^{ \pm a T}+B e^{b T}
$$

with $a, b>0$. We would study such possibility elsewhere [32].

\section{Conclusion and discussion}

We have studied moduli superpotentials with positive exponents. The corresponding scalar potentials have a quite rich structure. There are several local minima with different potential energies and a high barrier of $\mathcal{O}\left(M_{p}^{4}\right)$ as well as the runaway vacuum. This

\footnotetext{
${ }^{16}$ Then this potential is valid for $68.8 \pi / m_{3}<\operatorname{Re}(T)<68.8 \pi$.
} 
form of the scalar potentials has significant implications from the viewpoints of cosmology and particle phenomenology, e.g. the realization of inflation models, avoiding the overshooting problem and destabilization due to finite temperature effects. This type of potentials would be useful to realize the inflation and low-energy supersymmetry breaking. Thus, it would be interesting to study a new type of inflation models with positive exponent terms. In addition, we have shown that the modulus can be stabilized by a single term superpotential with positive exponent. That is one of the simplest models for the modulus stabilization and SUSY breaking. It would be interesting to apply this form of the potential to several phenomenological and/or cosmological aspects, e.g. the QCD axion.

\section{Acknowledgement}

H. A., T. H. and T. K. are supported in part by the Grand-in-Aid for Scientific Research No. 182496, No. 194494 and No. 20540266 from the Ministry of Education, Culture, Sports, Science and Technology of Japan. O. S. is supported by the MEC project FPA 2004-02015 and the Comunidad de Madrid project HEPHACOS (No. P-ESP-00346).

\section{References}

[1] S. B. Giddings, S. Kachru and J. Polchinski, Phys. Rev. D 66, 106006 (2002) arXiv:hep-th/0105097.

[2] S. Kachru, R. Kallosh, A. Linde and S. P. Trivedi, Phys. Rev. D 68, 046005 (2003) arXiv:hep-th/0301240.

[3] See, e.g., R. Kallosh, Lect. Notes Phys. 738, 119 (2008) arXiv:hep-th/0702059.

[4] R. Brustein and P. J. Steinhardt, Phys. Lett. B 302, 196 (1993) arXiv:hep-th/9212049.

[5] W. Buchmuller, K. Hamaguchi, O. Lebedev and M. Ratz, Nucl. Phys. B 699, 292 (2004) arXiv:hep-th/0404168]; W. Buchmuller, K. Hamaguchi, O. Lebedev and M. Ratz, JCAP 0501, 004 (2005) [arXiv:hep-th/0411109]; L. Anguelova, R. Ricci and S. Thomas, Phys. Rev. D 77, 025036 (2008) [arXiv:hep-th/0702168]; L. Anguelova and V. Calo, arXiv:0708.4159 [hep-th]; C. Papineau, arXiv:0802.1861 [hep-th].

[6] R. Kallosh and A. Linde, JHEP 0412, 004 (2004) [arXiv:hep-th/0411011.

[7] R. Kallosh and A. Linde, JHEP 0702, 002 (2007) arXiv:hep-th/0611183.

[8] H. Abe, T. Higaki and T. Kobayashi, Phys. Rev. D 73, 046005 (2006) arXiv:hep-th/0511160.

[9] K. Choi and K. S. Jeong, JHEP 0608, 007 (2006) arXiv:hep-th/0605108.

[10] K. Choi, K. S. Jeong, T. Kobayashi and K. i. Okumura, Phys. Rev. D 75, 095012 (2007) arXiv:hep-ph/0612258. 
[11] K. Choi, K. S. Jeong, T. Kobayashi and K. i. Okumura, Phys. Lett. B 633, 355 (2006) arXiv:hep-ph/0508029.

[12] S. Gukov, C. Vafa and E. Witten, Nucl. Phys. B 584, 69 (2000) [Erratum-ibid. B 608, 477 (2001)] arXiv:hep-th/9906070.

[13] R. Blumenhagen, B. Kors, D. Lust and S. Stieberger, Phys. Rept. 445, 1 (2007) arXiv:hep-th/0610327; D. Lust and S. Stieberger, Fortsch. Phys. 55, 427 (2007) arXiv:hep-th/0302221]; N. Akerblom, R. Blumenhagen, D. Lust and M. SchmidtSommerfeld, Phys. Lett. B 652, 53 (2007) arXiv:0705.2150 [hep-th]]; N. Akerblom, R. Blumenhagen, D. Lust and M. Schmidt-Sommerfeld, JHEP 0708, 044 (2007) arXiv:0705.2366 [hep-th]]; R. Blumenhagen and M. Schmidt-Sommerfeld, JHEP 0712, 072 (2007) arXiv:0711.0866 [hep-th]]; M. Billo, M. Frau, I. Pesando, P. Di Vecchia, A. Lerda and R. Marotta, JHEP 0710, 091 (2007) arXiv:0708.3806 [hepth]]; M. Billo, M. Frau, I. Pesando, P. Di Vecchia, A. Lerda and R. Marotta, JHEP 0712, 051 (2007) arXiv:0709.0245 [hep-th]].

[14] K. Choi and J. E. Kim, Phys. Lett. B 165, 71 (1985); L. E. Ibanez and H. P. Nilles, Phys. Lett. B 169, 354 (1986); E. Witten, Nucl. Phys. B 471, 135 (1996) arXiv:hep-th/9602070 ; T. Banks and M. Dine, Nucl. Phys. B 479, 173 (1996) |arXiv:hep-th/9605136]; K. Choi, Phys. Rev. D 56, 6588 (1997) arXiv:hep-th/9706171].

[15] D. Cremades, L. E. Ibanez and F. Marchesano, JHEP 0207, 009 (2002) arXiv:hep-th/0201205.

[16] D. Lust, P. Mayr, R. Richter and S. Stieberger, Nucl. Phys. B 696, 205 (2004) arXiv:hep-th/0404134;

[17] M. Bertolini, M. Billo, A. Lerda, J. F. Morales and R. Russo, Nucl. Phys. B 743, 1 (2006) arXiv:hep-th/0512067.

[18] M. Haack, D. Krefl, D. Lust, A. Van Proeyen and M. Zagermann, JHEP 0701, 078 (2007) arXiv:hep-th/0609211.

[19] J. F. G. Cascales and A. M. Uranga, JHEP 0305, 011 (2003) arXiv:hep-th/0303024; F. Marchesano and G. Shiu, Phys. Rev. D 71, 011701 (2005) arXiv:hep-th/0408059; F. Marchesano and G. Shiu, JHEP 0411, 041 (2004) [arXiv:hep-th/0409132].

[20] K. Choi, A. Falkowski, H. P. Nilles, M. Olechowski and S. Pokorski, JHEP 0411, 076 (2004) arXiv:hep-th/0411066.

[21] S. P. de Alwis, Phys. Lett. B 626, 223 (2005) arXiv:hep-th/0506266.

[22] H. Abe, T. Higaki and T. Kobayashi, Phys. Rev. D 74, 045012 (2006) arXiv:hep-th/0606095.

[23] H. Abe, T. Higaki and T. Kobayashi, Nucl. Phys. B 742, 187 (2006) arXiv:hep-th/0512232.

[24] R. Blumenhagen, M. Cvetic, F. Marchesano and G. Shiu, JHEP 0503, 050 (2005) arXiv:hep-th/0502095. 
[25] A. Saltman and E. Silverstein, JHEP 0411, 066 (2004) arXiv:hep-th/0402135; M. Gomez-Reino and C. A. Scrucca, JHEP 0605, 015 (2006) arXiv:hep-th/0602246]; O. Lebedev, H. P. Nilles and M. Ratz, Phys. Lett. B 636, 126 (2006) arXiv:hep-th/0603047.

[26] E. Dudas, C. Papineau and S. Pokorski, JHEP 0702, 028 (2007) arXiv:hep-th/0610297; H. Abe, T. Higaki, T. Kobayashi and Y. Omura, Phys. Rev. D 75, 025019 (2007) arXiv:hep-th/0611024]; arXiv:0801.0998 [hep-th]; O. Lebedev, V. Lowen, Y. Mambrini, H. P. Nilles and M. Ratz, JHEP 0702, 063 (2007) arXiv:hep-ph/0612035; M. Serone and A. Westphal, JHEP 0708, 080 (2007) arXiv:0707.0497 [hep-th]]; H. Abe, T. Higaki and T. Kobayashi, Phys. Rev. D 76, 105003 (2007) [arXiv:0707.2671 [hep-th]]; A. Achucarro and K. Sousa, arXiv:0712.3460 [hep-th]; H. Abe, T. Higaki, T. Kobayashi and Y. Omura, arXiv:0801.0998 [hep-th].

[27] H. Abe, T. Kobayashi and Y. Omura, JHEP 0711, 044 (2007) arXiv:0708.3148 [hep-th]].

[28] T. Higaki and T. Kobayashi, Phys. Rev. D 68, 046006 (2003) arXiv:hep-th/0304200.

[29] J. P. Conlon, JHEP 0605, 078 (2006) |arXiv:hep-th/0602233|; P. Svrcek and E. Witten, JHEP 0606, 051 (2006) [arXiv:hep-th/0605206]; K. Choi and K. S. Jeong, JHEP 0701, 103 (2007) arXiv:hep-th/0611279.

[30] J. J. Blanco-Pillado et al., JHEP 0411, 063 (2004) arXiv:hep-th/0406230.

[31] Z. Lalak, G. G. Ross and S. Sarkar, Nucl. Phys. B 766, 1 (2007) |arXiv:hep-th/0503178; A. Westphal, JCAP 0511, 003 (2005) [arXiv:hep-th/0507079]; J. P. Conlon and F. Quevedo, JHEP 0601, 146 (2006) arXiv:hep-th/0509012; J. J. Blanco-Pillado et al., JHEP 0609, 002 (2006) arXiv:hep-th/0603129]; P. Brax, A. C. Davis, S. C. Davis, R. Jeannerot and M. Postma, JCAP 0801, 008 (2008) arXiv:0710.4876 [hep-th]]; M. Badziak and M. Olechowski, arXiv:0802.1014 [hep-th].

[32] H. Abe, et al., in progress. 